International Journal of Bifurcation and Chaos, Vol. 22, No. 7 (2012) 1250168 (8 pages)

(C) World Scientific Publishing Company

DOI: $10.1142 / \mathrm{S} 0218127412501684$

\title{
ADAPTIVE GROWING NETWORKS COEVOLVING WITH THE SPREAD OF DISEASES
}

\author{
BENIAMINO GUERRA \\ Chair of System Design, ETH Zürich, 8092 Zürich, Switzerland \\ JESÚS GÓMEZ-GARDEÑES* \\ Departamento de Física de la Materia Condensada, \\ University of Zaragoza, Zaragoza 50009, Spain \\ Institute for Biocomputation and Physics of Complex Systems (BIFI), \\ University of Zaragoza, Zaragoza 50018, Spain \\ gardenes@gmail.com \\ VITO LATORA \\ School of Mathematical Sciences, Queen Mary, University of London, \\ Mile End Road, London E1 4NS, UK \\ Dipartimento di Fisica ed Astronomia, Università di Catania, \\ and INFN, Via S. Sofia 64, 95123 Catania, Italy \\ Laboratorio sui Sistemi Complessi, Scuola Superiore di Catania, \\ Via San Nullo 5/i, 95123 Catania, Italy
}

Received November 14, 2011; Revised February 20, 2012

\begin{abstract}
We study the evolution of an adaptive network whose growth occurs simultaneously to the propagation of a disease. The dynamics of the network growth is entangled to the spread of the disease, since the probability for a node in the network to get new links depends on its healthy or infected state. We analyze the influence that such coupling mechanism has both on the diffusion of the disease and on the structure of the growing networks. Our results point out that a strong interplay between network growth and disease spreading produces networks with degree-degree correlations and nontrivial clustering patterns.
\end{abstract}

Keywords: Complex networks; adaptive systems; epidemic spreading.

\section{Introduction}

In the last decade, the burst of studies in complex network science has spread across a large number of scientific disciplines [Albert \& Barabási, 2002; Newman, 2003]. These studies have mainly focused on the influence that the structure of interactions of large complex systems has on their functioning [Boccaletti et al., 2006]. In particular, most of the functional properties explored so far are related to the emergence of collective phenomena and their critical properties [Dorogovtsev et al., 2008], such as synchronization [Arenas et al., 2008], social phenomena [Szabó \& Fáth, 2007; Castellano et al., 2009], epidemics [Draief \& Massoulié, 2010] or congestion and jamming [Chen et al., 2012]. The extensive studies on the interplay between the structure and dynamics of complex networks have revealed the relevant role of the scale-free (SF) architecture of real complex networks, revealed from the powerlaw probability distribution of the number of contacts $(k)$ per individual $\left[P(k) \sim k^{-\gamma}\right]$, on the onset of the former collective phenomena.

\footnotetext{
${ }^{*}$ Author for correspondence
} 
More recently, the attention has focused on the study of adaptive complex networks [Gross \& Blasius, 2008; Perc \& Szolnoki, 2010; Herrera et al., 2011]. Once the implications that the structural properties of real networks have on their functioning have been intensively studied, the question about the origin of such structural patterns enters into play. This question is addressed by considering a dynamical evolution in which the structure of the network coevolves with the dynamical process at work. In this way, one entangles the evolution of the backbone of interactions with the dynamical state of the nodes that compose the network. These studies rely on two main approaches. On one hand, one can consider that the network has a fixed number of nodes whose ties adapt depending on the dynamical states of the node. This adaptive dynamics is thus based on rewiring processes or link plasticity, and it has been coupled with synchronization dynamics [Ito \& Kaneko, 2002; Zhou \& Kurths, 2006; Levina et al., 2007; Sorrentino \& Ott, 2008; Aoki \& Aoyagi, 2009; Assenza et al., 2011; Gutierrez et al., 2011], epidemic spreading processes [Gross et al., 2006; Han, 2007; Van Segbroeck et al., 2010; Marceau et al., 2010] or evolutionary games [Zimmermann et al., 2004; Pacheco et al., 2006; Li et al., 2007; Centola et al., 2007; Vazquez et al., 2007]. A second major line of research in adaptive networks focuses on the entanglement of the growth of the network and its dynamics. In this way, the formation of new links (launched from newcomers) depends on the dynamical state of each of the nodes that is already part of the growing network. However, once these links are formed they are not altered. We can find applications of such adaptive growth systems in synchronization [Sendiña-Nadal et al., 2008; Almendral et al., 2010] and evolutionary games [Poncela et al., 2008, 2009a, 2009b].

In this work, we study an adaptive network of the second kind, i.e. whose growth is coupled with the dynamical state of the system. In particular, we study a growing network that evolves simultaneously to the spread of a disease. The entanglement between growth and disease dynamics comes from the fact that the attachment probability of newcomers depends on the dynamical states (healthy or infected) of the nodes that are already present in the network, by favoring the attachment with those healthy nodes. Obviously, as the formation of new links (and thus the structure of the network) is affected by the spread of the epidemics, a feed-back loop is created in the system, and the dynamics of the spreading process is also affected by the way newcomers attach into the network. We will study the implications that this interplay between structure and dynamics has on both the impact of the epidemy and the structural network patterns that show up after the network is grown. Our results point out that a strong interplay may lead to a decrease in the epidemic impact while the structure of the network will show structural patterns such as degree-degree correlations and nontrivial patterns for the clustering coefficient.

\section{The Adaptive Network Model}

A previous work by some of the authors [Guerra \& Gómez-Gardeñes, 2010] introduced a simplified version of the following adaptive network model. However, in that study the focus was on the potentials of the annealed approach to study the dynamics on static and adaptive complex networks. Here, we will expand the adaptive model and focus on the results that the adaptive dynamics has on the structural and dynamical properties of the system.

The adaptive model we consider is the following. We start with a complete graph with $N(0)$ healthy nodes. At each time step, $t$, a new (also healthy) node with $m$ links is added to the network. The probability $\Pi_{j}(t)$ that one of the $N(t)$ nodes in the network at time $t$, say node $j$, receives one of the new $m$ links depends (in a manner that will be specified below) on whether the node is infected or not by an epidemic process running on the network. In fact, after $\tau$ time steps of the growth process, $5 \%$ of the $N(\tau)$ nodes in the network are infected. From this time on, the disease is propagated, namely one step of a Susceptible-Infected-Susceptible (SIS) epidemic model takes place after the addition of a new node to the network. In the SIS model the dynamical state of a node $j$, at a given time $t$, is a binary variable: healthy $s_{j}(t)=1$ or infected $s_{j}(t)=0$. Thus, macroscopically, the state of the population of $N(t)$ nodes at time $t$ can be described by the fraction $i(t)$ of infected individuals or, alternatively, by the fraction $(1-i(t))$ of healthy individuals. Following the rules of the SIS model, at each time step of the SIS dynamics, each node of the network will update its state as follows. Each healthy individual has a probability $\lambda$ of being infected by each of its infected neighbors. Thus, if a healthy individual, say node $j$, has a fraction $x_{j}(t)$ of its $k_{j}(t)$ neighbors 
in the infected state at time $t$, the probability that it will be infected at time $t+1$ is

$$
P_{j}^{h \rightarrow i}(t)=1-(1-\lambda)^{x_{j}(t) \cdot k_{j}(t)} .
$$

On the other hand, each infected individual, say $j$, at time $t$ has a constant probability $P_{j}^{i \rightarrow h}(t)=\mu$ of recovering, i.e. to come back to healthy state at the next time step $t+1$. In the SIS model, the relevant parameter that controls the spread of the disease is the ratio between infection and recovery rates, $\lambda / \mu$, while the impact of the disease of the population can be quantified by the fraction of infected individuals in the asymptotic limit, $i^{\infty}=\lim _{t \rightarrow \infty} i(t)$. In the classical version of the SIS model [Draief \& Massoulié, 2010], in which a well-mixed static set of $N$ individuals is considered, a phase transition for the order parameter $i^{\infty}$ occurs, so that, when $\lambda / \mu$ is below a certain critical value, the state of the system ends up in a free-of-disease state, $i^{\infty}=0$, while, when $\lambda / \mu$ is above the critical value, we have $i^{\infty}>0$ and the system is said to be in an epidemic state. It is also worth mentioning that when the SIS model is implemented on top of a static network (abandoning the well-mixed assumption) the epidemic onset takes place at $\lambda / \mu=\langle k\rangle /\left\langle k^{2}\right\rangle,\langle k\rangle$ and $\left\langle k^{2}\right\rangle$ being the first and the second moments of the degree distribution $P(k)$ of the network. For SF networks, $P(k) \sim k^{-\gamma}$, the second moment diverges when $\gamma<3$ (as happens in most real-world networks) and thus the epidemic onset goes to zero making SF networks extremely vulnerable to suffer epidemic states [Pastor-Satorras \& Vespignani, 2001].

In our adaptive network model, the spread of the SIS disease takes place on a growing system. Moreover, the network growth depends on the health of the infected state of the nodes. In fact, the probability that a node $j$ receives a link from a new node at time $t, \Pi_{j}(t)$ is defined as:

$$
\Pi_{j}(t)=\frac{k_{j}^{\alpha}(t)\left[1-\epsilon+\epsilon \cdot s_{j}(t)\right]}{\sum_{l=1}^{N(t)} k_{l}^{\alpha}(t)\left[1-\epsilon+\epsilon \cdot s_{l}(t)\right]} .
$$

Where $\alpha$ and $\epsilon$ are two parameters ranging in the interval $[0,1]$. The exponent $\alpha$ tunes the importance given to the degree of node $j$. In fact, $\alpha=0$ corresponds to no-dependence on the node degree, while $\alpha=1$ is the usual linear preferential attachment of the Barabási and Albert growth model [Barabási \& Albert, 1999]. On the other hand, the parameter $\epsilon$ measures the importance given to the disease state of the network: for $\epsilon=0$ there is no interplay between SIS and network dynamics. In this case, the dynamics of the disease and the network growth are completely independent, and we obtain networks with an exponentially decaying degree distribution $P(k) \sim \mathrm{e}^{-k}$, when $\alpha=0$, and SF networks with $P(k) \sim k^{-3}$ for $\alpha=1$. On the contrary, as $\epsilon \rightarrow 1$ new nodes tend to increasingly avoid linking to infected individuals.

\section{Results}

Let us first study how the incidence of the disease changes as function of the two parameters $\alpha$ and $\epsilon$, tuning, respectively, the dependence on the node degree and the node internal state. In Fig. 1, we report the fraction of healthy individuals in the stationary state, $s^{\infty}=\left(1-i^{\infty}\right)$, typically achieved when the network size has reached $N(t) \sim 10^{3}$. We have set three different values of the infection rate: $\lambda=0.07,0.12$ and 0.3 . From the figure, it is clear that when the selection of newcomers becomes more biased towards large degree nodes $(\alpha \rightarrow 1)$, the impact of the SIS disease increases, regardless of the value of $\epsilon$. The reason behind this behavior is clearly that preferential attachment increases the connectivity of the individuals with a large number of contacts and thus reinforces the ability of the SIS disease to spread across the population. However, for a fixed value of $\alpha$, we observe that increasing the tendency of newcomers to connect with healthy nodes, i.e. increasing the interplay between growth and SIS dynamics, produces an effective way for decreasing the impact of the disease.

We next focus on the more interesting issue about the structural patterns produced by adaptivity in the strong interplay limit $(\epsilon \rightarrow 1)$. In Fig. 2 we show the degree distributions $P(k)$ produced for different values of the infection rate $\lambda$ in the two cases $\alpha=0$ and $\epsilon=0.99$ (left panel), and $\alpha=1$ and $\epsilon=0.99$ (right panel). As expected, when $\alpha=0$, the networks produced have exponentially decaying degree distributions $P(k)$. However, a careful check of the tails of these distributions show a dependence on the value of the infection rate $\lambda$. To show more clearly this latter dependence we have plotted in the inset of the figure the evolution of the second moment, $\left\langle k^{2}\right\rangle$, of the degree distribution as $\lambda$ increases. The second moment measures the heterogeneity of the degree distributions as it is very sensitive to the behavior of their 



Fig. 1. The contour plots show the asymptotic fraction of healthy individuals, $s^{\infty}$, as a function of the parameters $\alpha$ and $\epsilon$ controlling the preference of newcomers towards connected and healthy nodes respectively [see Eq. (2)]. From left to right, the values of the infection rate $\lambda$ are $0.07,0.12$ and 0.3 . In all cases, the recovery rate is fixed to $\mu=1$, while the final network size is $5 \cdot 10^{3}$ nodes. From the plots, it is clear that the incidence of the disease decreases as $\alpha$ grows, and it decreases as $\epsilon$ increases.

tails. From the inset we observe that as $\lambda$ increases, the distributions become more homogeneous [shortening the tails of $P(k)$, reaching a minimum value around $\lambda \sim 0.2$. From this value on, the tails start to enlarge, reaching the exponential behavior again for large values of $\lambda$. The minimum found for $\left\langle k^{2}\right\rangle$ occurs close to the epidemic onset, when the number of infected nodes is relatively small (compared to the number of nodes in the system) and they are mainly placed on the most connected nodes of the network. Therefore, in the strong interplay limit $(\epsilon=0.99)$ this hierarchical placement avoids these latter nodes to capture new links thus narrowing the exponential degree distribution. For large values of $\lambda$ the disease reaches almost all the population and newcomers do not distinguish between connectivity classes, thus making again the attachment at random and recovering the exponential decay for $P(k)$.
This evolution is more evident in the case of $\alpha=1$ (right panel in Fig. 1). In this case, the degree distribution behaves as a power law $P(k) \sim k^{-3}$ for large values of $\lambda$ (high impact of the disease). However, close to the epidemic onset the tails of $P(k)$ become stretched because of the hierarchical placement of infected nodes in large degree nodes which, for $\alpha=1$, correspond to hubs with an extremely large number of connections. This homogenization becomes again evident from the inset of the figure in which the evolution $\left\langle k^{2}\right\rangle(\lambda)$ shows a minimum for low values of $\lambda$.

Now we focus on the patterns obtained for the clustering coefficient. The clustering coefficient of a node, say $j$, with degree $k_{j}$ is defined as the number of connections among the neighbors of $j$ divided by the maximum possible number of these neighborneighbor links: $k_{j} \cdot\left(k_{j}-1\right) / 2$. In Fig. 3, we show
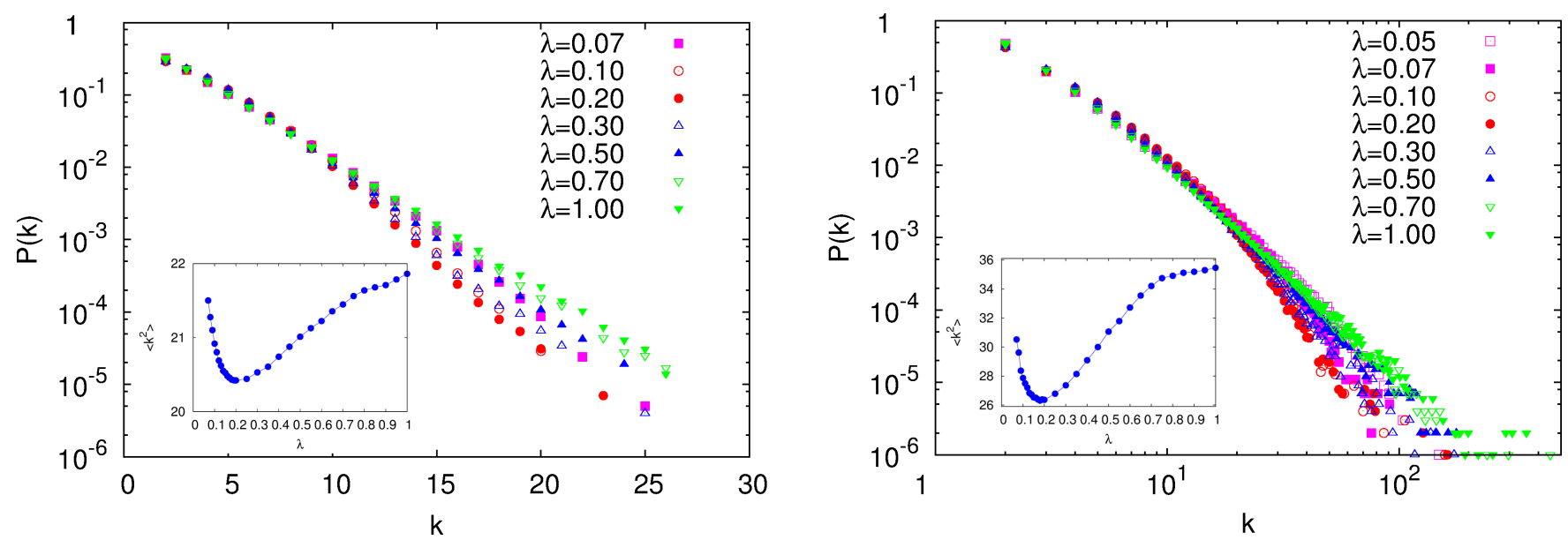

Fig. 2. Degree distributions $P(k)$ of the adaptive networks in the strong coupling growth-disease $(\epsilon=0.99)$ and for several values of the infection rate $\lambda$. The plot on the left refers to the case $\alpha=0$ while the plot on the right refers to $\alpha=1$. The insets show the evolution of the second moment $\left\langle k^{2}\right\rangle$ of the degree distribution as $\lambda$ increases. As in Fig. 1 the value of the recovery rate is set to $\mu=1$ and the final size of the networks is $5 \cdot 10^{3}$ nodes. 

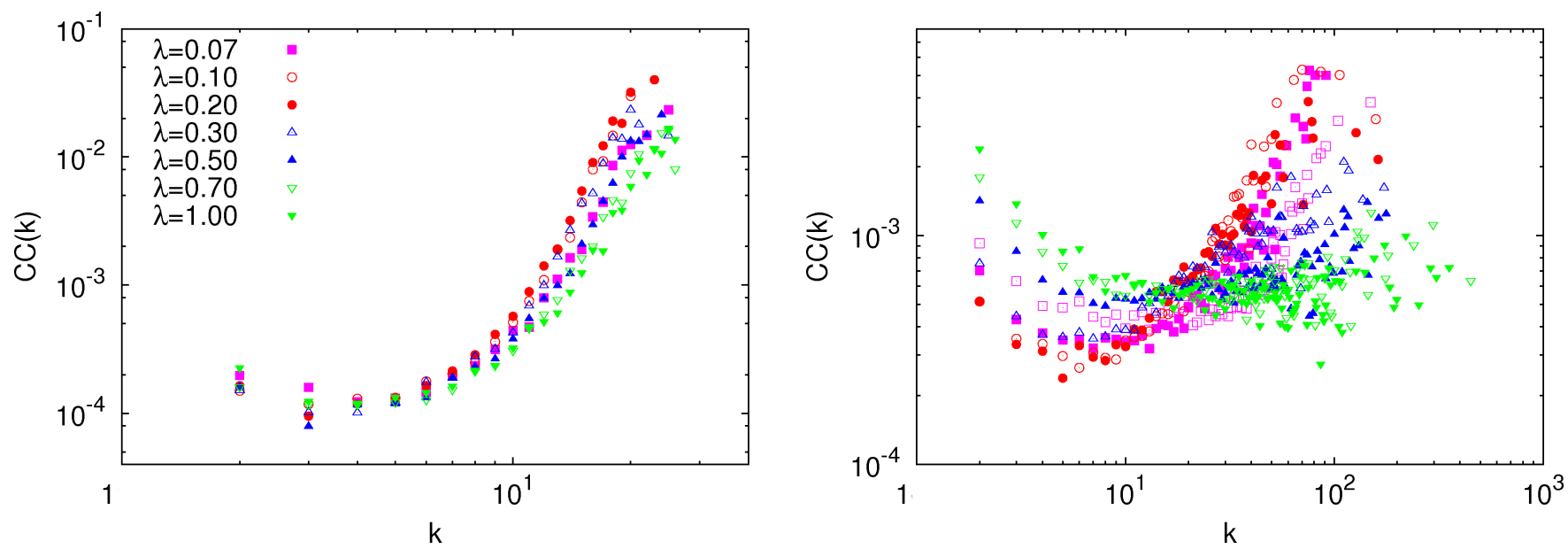

Fig. 3. Clustering coefficient as a function of the degree of the nodes $\mathrm{CC}(k)$ of the adaptive networks in the strong interplay limit $(\epsilon=0.99)$ and several values of the infection rate $\lambda$. The plot on the left is for the situation with $\alpha=0$ while $\alpha=1$ for those curves on the right panel. As in Fig. 1 the value of the recovery rate is set to $\mu=1$ and the final size of the networks is $5 \cdot 10^{3}$ nodes.

the value of the clustering coefficient as a function of the degree $k$ of the nodes, $\mathrm{CC}(k)$. To this end we have averaged the clustering of the nodes having the same number of neighbors as

$$
\mathrm{CC}(k)=\frac{k(k-1)}{2 N P(k)} \sum_{\left\{j \mid k_{j}=k\right\}} \sum_{l, m} \frac{A_{j l} A_{j m}}{2},
$$

where $\left\{A_{j l}\right\}$ is the adjacency matrix of the network defined as $A_{j l}=1$ when nodes $j$ and $l$ are connected while $A_{j l}=0$ otherwise. The plots in Fig. 2 correspond to the strong interplay limit $(\epsilon=1)$ while the left panel is for $\alpha=0$ and $\alpha=1$ for the right one. After the results discussed in Fig. 2 we can take the curves $\mathrm{CC}(k)$ for $\lambda=1$ (large impact of the disease) as references since, from what we discussed above, the large degree of infection of the evolving networks masks the newcomers' preference for attaching to healthy individuals while $\alpha$ (controlling the preference towards high degree nodes) turns out to be the dominant parameter. In this limit $(\lambda=1)$ we observe that for $\alpha=0$ the clustering increases with the degrees of nodes. However, for smaller values of $\lambda$ the same trend is observed. On the contrary, $\mathrm{CC}(k)$ patterns for the case of $\alpha=1$ are much more sensitive to changes in the infection rate $\lambda$ at work. For $\lambda=1$ we observe a rather constant pattern for $\mathrm{CC}(k)$ pointing out that clustering is not dependent on the degree hierarchy of nodes. However for small $\lambda$ values, specially for those around the minimum of $\left\langle k^{2}\right\rangle$ in Fig. 2, we observe a strong positive correlation between clustering and degree.
We round off our analysis by checking the evolution of the degree-degree correlations, i.e. evaluating the relation between the degrees of connected nodes. To this aim, we measure the average degree of the neighbors of nodes of degree $k, k_{n n}(k)$ which is defined as:

$$
k_{n n}(k)=\frac{1}{k N P(k)} \sum_{\left\{j \mid k_{j}=k\right\}} \sum_{l} A_{j l} k_{l} .
$$

By measuring the trend of the function $k_{n n}(k)$ we can distinguish between assortative networks (for which the $k_{n n}(k)$ is an increasing function and thus connections are more likely to be established between nodes of identical degrees), disassortative networks (for which the $k_{n n}(k)$ is a decreasing function and links are used to connect nodes of different degrees) and networks with no degree-degree correlations (for which the $k_{n n}(k)$ are nearly constant so that there is no relation between the degrees of neighboring nodes). In Fig. 4 we observe again the correlation patterns in the strong interplay limit $(\epsilon=0.99)$ for $\alpha=0$ (left panel) and $\alpha=1$ (right panel). Taking again the curves corresponding to $\lambda=1$ as references, we observe that when the disease is close to the epidemic onset there is a tendency towards assortativity, i.e. the slope of the curves $k_{n n}(k)$ increases with respect to those found for $\lambda=1$. This tendency towards assortativity is more evident in the case of $\alpha=1$. In this case, the slope for $\lambda=1$ is slightly negative as expected from a SF network constructed with the Barabási-Albert model. However, for low 

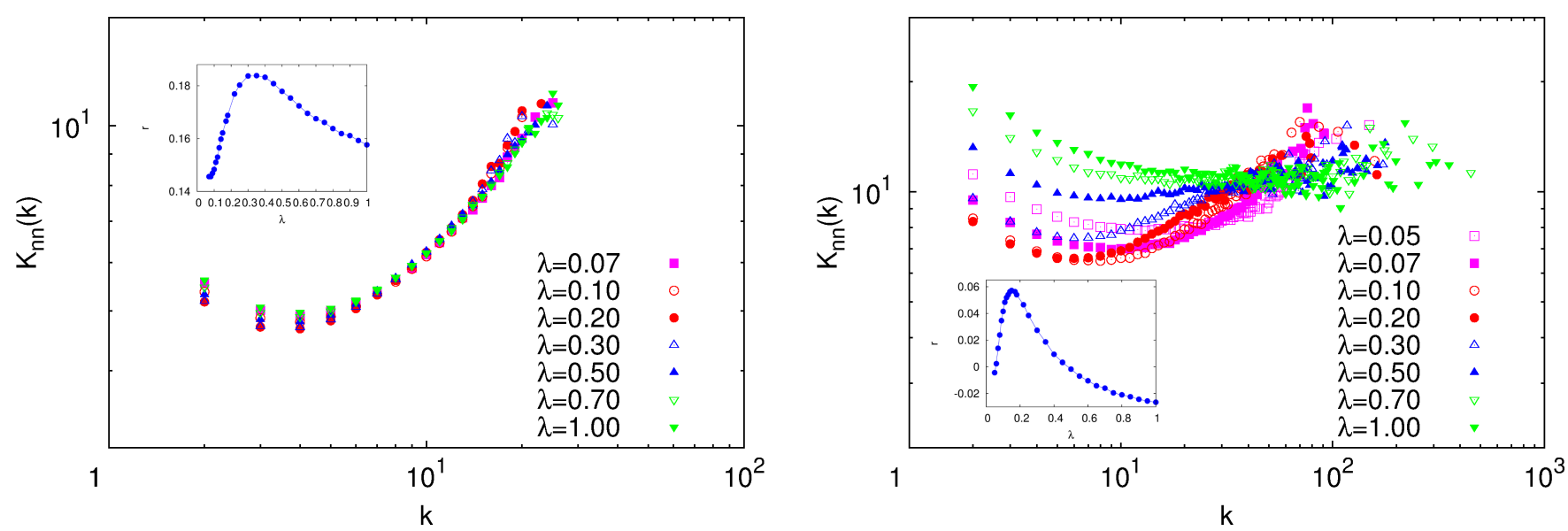

Fig. 4. Average degree of the neighbors of nodes with degree $k, k_{n n}(k)$, of the adaptive networks in the strong interplay limit $(\epsilon=0.99)$ and several values of the infection rate $\lambda$. The plot on the left is for the situation with $\alpha=0$ while $\alpha=1$ for those curves on the right panel. The insets show the evolution of the assortativity index $r$ of the networks as $\lambda$ increases. As in Fig. 1 the value of the recovery rate is set to $\mu=1$ and the final size of the networks is $5 \cdot 10^{3}$ nodes.

$\lambda$ values the degree-degree correlations turn positive and the assortative character of the adaptive networks is remarkable. This tendency is again supported from the large degree of infection of the hubs for this low value of $\lambda$. This state prevents many of the connections with newcomers (low degree nodes) thus making more relevant those connections previously established with other large degree nodes. On the contrary, many connections between poorly connected (and thus healthy) nodes are favored, so that the average degree of the neighbors of nodes with a small degree turns out to be also small. The evolution of the assortative character of networks is summarized in the insets of both panels in Fig. 4 by computing the assortativity index, $r$, introduced in [Newman, 2002] which takes positive (negative) values for assortative (disassortative) networks.

\section{Conclusions}

In this work we have analyzed an adaptive complex network in which two dynamical processes evolve entangled. These two dynamics are the growth of the network (with the addition of new nodes into the backbone) and an SIS disease dynamics (so that nodes can be in two dynamical states: healthy or infected). The two dynamical processes are entangled via the way newcomers are attached to those nodes that already take part in the evolving network. In this way, we have assumed that when the growth and SIS dynamics are strongly correlated newcomers tend to avoid infected individuals. Besides, we have also considered the possible preference of newcomers towards large degree nodes. By solving numerically this adaptive model we have obtained several results. First, we have shown that the strong interplay between the two dynamical processes decreases the impact of the disease on the growing network due to the ability of newcomers to avoid infected individuals. However, this ability (when the degree of infection is not very high) has important consequences on the final structure of the network since those individuals that are more likely infected are the more connected ones. Therefore, those adaptive systems evolved in the large interplay regime evolve towards more degree-homogeneous backbones than what was expected if the SIS dynamics and the growth processes were uncorrelated. Additionally these networks show clustering-degree and degreedegree correlations. In particular, the decrease of low-to-large degree connections (made from the attachment of newcomers to hubs) when the disease is close to the epidemic onset favor the onset of positive correlations for both the clustering-degree and the degree-degree correlations. These results support the importance of considering the adaptive networks perspective in order to explain both the plasticity of the network backbone as a function of the dynamical regimes it can explore, and the consequences that this plasticity has on the functioning of the adaptive network.

\section{Acknowledgments}

This work has been supported by the Spanish DGICYT under projects FIS2008-01240, FIS201125167 and MTM2009-13848, and by the Comunidad de 
Aragón through Project No. FMI22/10. B. Guerra acknowledges partial support from the Swiss State Secretariat for Education and Research SER C09.0055. J. Gómez-Gardeñes is supported by MICINN through the Ramón y Cajal program.

\section{References}

Albert, R. \& Barabási, A.-L. [2002] "Statistical mechanics of complex networks," Rev. Mod. Phys. 74, 47.

Almendral, J. A., Leyva, I., Sendiña-Nadal, I. \& Boccaletti, S. [2010] "Interacting oscillators in complex networks: Synchronization and the emergence of scalefree topologies," Int. J. Bifurcation and Chaos 20, 753.

Aoki, T. \& Aoyagi, T. [2009] "Co-evolution of phases and connection strengths in a network of phase oscillators," Phys. Rev. Lett. 102, 034101.

Arenas, A., Díaz-Guilera, A., Kurths, J., Moreno, Y. \& Zhou, C. [2008] "Synchronization in complex networks," Phys. Rep. 469, 93.

Assenza, A., Gutierrez, R., Gómez-Gardeñes, J., Latora, V. \& Boccaletti, S. [2011] "Emergence of structural patterns out of synchronization in networks with competitive interactions," Scient. Rep. 1, 99.

Barabási, A. L. \& Albert, R. [1999] "Emergence of scaling in random networks," Science 286, 509.

Boccaletti, S., Latora, V., Moreno, Y., Chavez, M. \& Hwang, D. U. [2006] "Complex networks: Structure and dynamics," Phys. Rep. 424, 175-308.

Castellano, C., Fortunato, S. \& Loreto, V. [2009] "Statistical physics of social dynamics," Rev. Mod. Phys. 81, 591.

Centola, D., González-Avella, J. C., Eguíluz, V. M. \& San Miguel, M. [2007] "Homophily, cultural drift, and the co-evolution of cultural groups," J. Conf. Resol. $\mathbf{5 1 ,} 905$.

Chen, S., Huang, W., Cattani, C. \& Altieri, G. [2012] "Traffic dynamics on complex networks: A survey," Math. Probl. Engin. 2012, 732698.

Dorogovtsev, S. N., Goltsev, A. V. \& Mendes, J. F. F. [2008] "Critical phenomena in complex networks," Rev. Mod. Phys. 80, 1275.

Draief, M. \& Massoulié, L. [2010] Epidemics and Rumours in Complex Networks (Cambridge University Press, Cambridge, UK).

Gross, T., Dommar D'Lima, C. \& Blasius, B. [2006] "Epidemic dynamics on an adaptive network," Phys. Rev. Lett. 96, 208701.

Gross, T. \& Blasius, B. [2008] "Adaptive coevolutionary networks: A review," J. R. Soc. Interf. 5, 259.

Guerra, B. \& Gómez-Gardeñes, J. [2010] "Annealed and mean-field formulations of disease dynamics on static and adaptive networks," Phys. Rev. E 82, 035101 .
Gutierrez, R., Amann, A., Assenza, A., GómezGardeñes, J., Latora, V. \& Boccaletti, S. [2011] "Emerging meso- and macroscales from synchronization of adaptive networks," Phys. Rev. Lett. 107, 234103.

Han, X.-P. [2007] "Disease spreading with epidemic alert on small-world networks," Phys. Lett. A 365, 1.

Herrera, J. L., Cosenza, M. G., Tucci, K. \& GonzálezAvella, J. C. [2011] "General coevolution of topology and dynamics in networks," EPL 95, 58006.

Ito, J. \& Kaneko, K. [2002] "Spontaneous structure formation in a network of chaotic units with variable connection strengths," Phys. Rev. Lett. 88, 028701.

Levina, A., Herrmann, J. M. \& Geisel, T. [2007] "Dynamical synapses causing self-organized criticality in neural networks," Nature Phys. 3, 857.

Li, W., Zhang, X. \& Hu, G. [2007] "How scale-free networks and large-scale collective cooperation emerge in complex homogeneous social systems," Phys. Rev. E 76, 045102.

Marceau, V., Noel, P.-A., Hébert-Dufresne, L., Allard, A. \& Dubé, L. J. [2010] "Adaptive networks: Coevolution of disease and topology," Phys. Rev. E 82, 036116.

Newman, M. E. J. [2002] "Assortative mixing in networks," Phys. Rev. Lett. 89, 208701.

Newman, M. E. J. [2003] "The structure and function of networks," SIAM Rev. 45, 167.

Pacheco, J. M., Traulsen, A. \& Nowak, M. A. [2006] "Coevolution of strategy and structure in complex networks with dynamical linking," Phys. Rev. Lett. 97, 258103.

Pastor-Satorras, R. \& Vespignani, A. [2001] "Epidemic spreading in scale-free networks," Phys. Rev. Lett. 86, 3200 .

Perc, M. \& Szolnoki, A. [2010] "Coevolutionary games A mini review," BioSyst. 99, 109.

Poncela, J., Gómez-Gardeñes, J., Floría, L. M., Sánchez, A. \& Moreno, Y. [2008] "Complex cooperative networks from evolutionary preferential attachment," PLoS ONE 3, e2449.

Poncela, J., Gómez-Gardeñes, J., Moreno, Y. \& Traulsen, A. [2009a] "Evolutionary game dynamics in a growing structured populations," New J. Phys. 11, 083031.

Poncela, J., Gómez-Gardeñes, J., Floría, L. M., Moreno, Y. \& Sánchez, A. [2009b] "Cooperative scale-free networks despite the presence of defector hubs," EPL 88, 38003.

Sendiña-Nadal, I., Buldú, J. M., Leyva, I. \& Boccaletti, S. [2008] "Phase locking induces scale-free topologies in networks of coupled oscillators," PLoS ONE 3, e2644.

Sorrentino, F. \& Ott, E. [2008] "Adaptive synchronization of dynamics on evolving complex networks," Phys. Rev. Lett. 100, 114101. 
Szabó, G. \& Fáth, G. [2007] "Evolutionary games on graphs," Phys. Rep. 446, 97.

Van Segbroeck, S., Santos, F. C. \& Pacheco, J. M. [2010] "Adaptive contact networks change effective disease infectiousness and dynamics," PLoS Comp. Biol. 6, e1000895.

Vazquez, F., González-Avella, J. C., Eguíluz, V. M. \& San Miguel, M. [2007] "Time-scale competition leading to fragmentation and recombination transitions in the coevolution of network and states," Phys. Rev. E 76, 46120.

Zhou, C. \& Kurths, J. [2006] "Dynamical weights and enhanced synchronization in adaptive complex networks," Phys. Rev. Lett. 96, 164102.

Zimmerman, M. G., Eguíluz, V. M. \& San Miguel, M. [2004] "Coevolution of dynamical states and interactions in dynamic networks," Phys. Rev. E 69, 065102. 